\title{
JournalScan
}

\section{Exploring the motivation of patients and professionals and ways in which information might support a change in the relationships between them

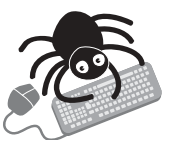

Please visit the QSHC website (http://www.qshc.com) for links to these articles - many to full text.

The papers in this issue of JournalScan are divided into two categories: the first looks at the often implicit motivations of providers, professionals, and patients and the differences between them; and the second considers ways in which information is and might be used to improve relationships between them.

\section{Exploring the motivation of providers, professionals and patients}

Differences in the motivation of providers $\rightarrow$ A paper in Social Science and Medicine explores the motivations of managers and doctors in UK hospitals. It highlights a lack of homogeneity among managerial motivations, but also some commonality between those working closest to service provision. The literature review showed that doctors and managers are key actors in hospitals and that "much of the organisational change within the UK

.. has been an attempt to redefine the balance of power [between them], i.e. to strengthen the role of management and to encourage participation by doctors in management".

The researchers constructed four hypotheses for empirical testing "based on the premise that doctors and managers have different sets of objectives in accordance with their incentives structures and professional duties":

- Doctors aim to expand their service.

- Managers aim to work within the financial regime by meeting the break even constraint.

- In "good times" [as financially defined] these objectives will converge to form a Trust objective of service expansion subject to a break even constraint.

- In "bad times" the objectives of doctors and managers will diverge.

The paper draws on a survey of 1500 consultants (including chairs of directorates and those on the board) and managers (CEOs, board members, service managers and clinical professionals). Three main questions in the survey assessed motivation.

(1) To what extent does your own career depend on the following factors (responses included financial success, speciality success, your individual reputation, the reputation of the hospital, the standard of $R \& D$, and continued provision of your clinical service)? (2) Within the trust, how would you rank the priorities for the following sets of people (same variables as in question 1)?

(3) How have priorities changed since the organisation became a trust?

Unsurprisingly, both managers and doctors regarded personal reputation as the most important factor in their career but, while managers thought financial success was very important, doctors thought this low in importance. There were differences in all other variables, the smallest difference being in continued provision of clinical service which was regarded as important by both groups.

In answer to question 2, consultants - as might be imaginedsaid managers were mostly interested in financial break even. More interesting results came from managerial responses and the differences between them: "Service/business managers and clinical managers gave highest priority to maintaining quality, while the corporate groups of CEO, board directors and other corporate managers ranked financial break even first. Managers closest to the point of service delivery thus made the surprising assertion here that maintaining quality was more important to them than financial break even". Managers gave more importance to quality for themselves than they would guess others would, "taking the view that they had a more conscientious approach to quality than had most of their peers". The modal response among consultants to question 3 was, for the most part, "no change", while the modal response among managers was "stronger" in all categories.

The results appear to confirm that doctors and managers are motivated by different goals; they responded overall as "two independent groups". They show that "if doctors and managers are to avoid conflicts of interest, it follows that the government will need to continue to invest and provide growth money to the acute sector to maintain organisational stability".

"The introduction of quasi-market reform in the UK through the purchaser/provider split was originally envisaged as a mechanism to bring about greater responsiveness ... to financial incentives". But "the empirical evidence of this study ... indicates that exposure to management does not produce a marked change in doctors' motivation". Rather, the reverse appears to be true. Closer proximity between hospital consultants and managers in a decentralised organisational structure seems to shift the perceptions and aspirations of managers, producing a gap between the motivation of those who are close to consultants and those who are set apart at the strategic apex of the organisation. "Professionals have not been captured by the organisation; rather, the organisation is dominated by professionals and motivated by professional goals".

A Crilly T, Le Grand J. The motivation and behaviour of hospital trusts. Soc Sci Med 2004;58:1809-23.

Professionals and patients: striving for autonomy and detachment A paper with a sociological bent explores the motivation of professionals and patients in surgical rehabilitation in a 1 year study of ethnographic fieldwork in a neurosurgical clinic in Portugal. It argues that professionals seek autonomy in patients as a sign of successful recovery while patients seek detachment from the surgical team.

"The experience of illness refers not only to physical suffering but also to shifts in the social placement of the person, which affects how the person interprets situations and his/her position in them". "In surgery, patients are confronted with one main question: whether or not to give over power in their lives to surgeons, surgery and its material apparatuses in order to regain the aspects of the self that were affected by illness."

Patients undergoing surgery temporarily lose their sense of selfthat is, their independence - and during the process of rehabilitation reconstitute this with the help of the clinical team who aim for a sense of autonomous self in patients. The research is concerned with "the strategies used to reconstruct the self" - to detach themselves from medical support, "undoing the link between surgeon and patient". "Repersonification corresponds to the public recognition of a patient's ability to take care of themselves." The effort to achieve this is made by the clinical team because "patients arrive at the wards from the operating room lacking the main characteristic of personhood as it is defined on the neurosurgical wards: autonomy".

Two cases are presented in whom "the practices in particular divisions of the surgical unit (the operating room and its immediate surrounds), the ITU, the wards, laboratory and outpatient clinic" were observed.

The first patient spent most of the 2 days after his operation sleeping. The researcher was struck by the "intensity of the work on and around him: repeated interactions, measurements of 
temperature and blood pressure, surveillance of the wounds and their dressing, requests to express pain and its locations, rigorous management of drug taking routines and intense spoken and written commentary on the patient's body and actions and reactions to nursing work" ${ }^{\prime \prime}$. With the exception of blurred vision (which worried him but not the doctors), the patient was fine and free of infection. The author argues that it is the movement from dependence to independence that is critical.

The second patient spent a difficult 2 weeks after his operation regaining independence. His "alternation between relative autonomy and dependence" made it difficult to plan his care. After a further relapse he was taken to the ICU. His relatives were startled "by the different environment in which they found him, breathing with the help of a ventilator, his heartbeat measured by a machine" He died on the night after his transfer. The author considers that "the ward work can be seen as a set of procedures for moving patients from one arrangement of material and social relations to the next" When patients become autonomous "it is possible to negotiate discharge". "In the coming together of ward accounts and scientific representations and measurements, detachment is accomplished through the possibility of representing the collective as coherently moving towards autonomy from the people, devices and techniques to which it was attached before"

In contrast to sociologists who argue that medical interventions disempower, the author argues that scientific and technical processes seek to support a patient's search for autonomy and rediscovery of self.

A Moreira T. Self, agency and the surgical collective: detachment. Sociol Health IIIn 2004;26:32-49.

Providers are encouraged to treat people with dignity, but what does it look like? "National and international policies for the health and social care of older people increasingly emphasise the right to and the need for dignity." In England, new core standards on which providers' performance will be assessed include a requirement to treat people with dignity and respect. The researchers cite evidence that this can impact positively on treatment and its absence can have an economically detrimental effect on providers because of increased complaints. Yet, despite these findings, "there is evidence of undignified treatment in health and social situations, especially in relation to older age groups".

In turning aspirations into practice, one problem is that "the term 'dignity' is rarely defined and little empirical research has been undertaken in relation to older people's experience of dignity". The authors set out to explore the meaning of dignity from the perspectives of older people. Fiffeen focus groups involving a total of 72 people were held in cities in South West England and South Wales.

"There was strong evidence to suggest that dignity was salient to the concerns of older people. However, it was a problematic concept in that it was easier for people to talk about its absence". Three main categories emerged from the analysis:

- identity;

- human rights; and

- autonomy.

Identity (in the sense of how dignity affects the self) was the category with the largest number of themes attached to it. One participant said if people "lost their dignity within themselves, they no longer think they are a person that matters". Examples of how people are stripped of their sense of self include: being excluded from conversations, a lack of attention to people's appearance by hospital or residential staff such as haphazard buttoning of clothes, or the exposure of bodies - for instance, when a hoist was used for lifting. Participants mentioned disrespectful labels attached to old age such as "bed blockers" and "geriatrics". Even simple forms of address can enhance or jeopardise dignity-for example, many older people do not like being addressed by their forename.

In terms of human rights, a key issue that emerged was euthanasia. People want to die with dignity. "I had no choice about being brought into the world but I hope and want a choice about how I should depart from it". It was thought that, due to advanced medical interventions, death had become medicalised. "I think we are a death denying society, but I personally feel that death is natural, when it's time to die just go, why keep prolonging it, when doctors are taking power, they control too much, even death is medicalised"

Autonomy was the third key issue in the sense of individuals having "capacity for independence and autonomy". Some felt that professionals stripped people of autonomy in the way they treated them, such as social workers. "They want you to do exactly what they want you to do and that's what I have really objected to ... they are taking my choice away, they seem to want to take your choice away, they don't want you to have an opinion, they want to tell you 'you will wear pads', you will go to a day care centre"'

The authors consider that "at a time when notions of choice, empowerment and consumerism are high on policy agendas, there is clearly room for considerable improvement in current practice". According to participants, professionals who appear to have little time for such quality issues are not currently addressing these important aspects of dignified care.

$\Delta$ Woolhead G, Calnan M, Dieppe P, et al. Dignity in older age: what do older people in the United Kingdom think? Age Ageing 2004;33:165-70.

What dimension of patient satisfaction most increases the likelihood of them returning to a provider? Datient satisfaction is often assumed to be a virtue in and of itself and, in addition, is often used as a proxy measure for quality of care. It is assumed that satisfaction will increase the likelihood of a patient returning to the provider. However, while these assumptions have intuitive appeal, there is surprisingly little data to justify them.

In testing this assumption, Garman et al examined whether 1485 patients who completed a satisfaction survey in 1997 returned in the following 2 year period (without making the point that such a choice may not be entirely positive). The researchers explored 10 dimensions and 47 indicators corresponding to satisfaction with admissions, nursing staff, physicians, meals, room, ancillary services, technicians, visitor services, discharge and an overall total score.

"Overall satisfaction as measured by averaging all 47 indicators turned out to be a weaker predictor than an empirically derived subscale consisting of just seven items, suggesting that the majority of the satisfaction items did not add significant incremental value in predicting return-to-provider behaviour".

The important variables were courtesy, information from nurses regarding tests, information from physicians regarding family, time physicians spent with patient, physicians' information regarding treatments, physicians' concern regarding questions, and the adequacy of information given to the family. Two factors were especially important: (1) how well care providers explained and informed patients regarding their medical procedures, and (2) the level of concern shown by care providers towards patients and their families.

The authors consider that, in terms of satisfaction surveys, it may be that the survey may not be asking all of the "right" questionsthat is, it may not be fully tapping the areas important to patients in making decisions about their care providers. The results suggest "that the goal of customer returns would be best served by focusing on provider responsiveness to patients and families, particularly in terms of patient education, rather than on improving other aspects of service (such as the quality of food or appearance of the hospital)".

A Garman A, Garcia J, Hargreaves M. Patient satisfaction as a predictor of return-to-provider behaviour: analysis and assessment of financial implications. Qual Manag Health Care 2004;13:75-80. How can information be used to improve
relationships between providers, patients
and professionals? ways of accessing health information and healthcare advice. The following papers explore the capacity for information to further develop relationships between patients, providers, and professionals. Each suggests that there is a long way to go before information is able to play the positive role many argue it can.

What capacity is there for alternative forms of consultation in primary care by telephone and email? - Telephone consulting is used mainly in primary care for out of hours work and for triage. A team of researchers set out to determine, for a consecutive sample of adult patients consulting their 
GP, whether their consultation could have been managed over the telephone or by email.

The study was conducted in one group practice in Leeds with eight GPs over a 2 week period in July and August 2002. All consecutively attending adult patients were asked to complete a questionnaire relating to their consultations and GPs were asked to complete similar sheets. The participants were also asked to categorise the consultation into a new problem, a planned follow up or review of an existing problem, a medication review, a further consultation because a previous problem was not settled, or another reason. Data were collected from 1150 consultations. Paired data (forms from patients and GPs) were available for 711 .

Interestingly, "GPs thought that a far greater proportion of these consultations could have been dealt with by telephone $(22.8 \%)$ or email $(17.6 \%)$ than did patients $(9.6 \%$ and $5.8 \%$ respectively)" Patients value face to face contact more than GPs and are more wary of change. They fear that exchange in any other form will be limited. The paper concludes that, while there may be scope for alternative consultations, patients may need more convincing that this is acceptable. The consultation category where there may be scope for change is medication review. Almost $17 \%$ of patients thought these could be done by telephone and $14 \%$ by email.

$\Delta$ Neal R, Pascoe S, Allgar V. Alternative forms of consulting: survey of patients and GPs about their consultations. Fam Pract 2004;21:140-2.

The conundrums of searching for health information on the internet Consumers and patients are confronted with a plethora of information about health and health care, especially through the proliferation of web based information. They often have difficulty, however, in identifying and accessing answers to their idiosyncratic health questions using standard information retrieval techniques. This paper focuses on health information retrieval via the internet that is initiated by consumers to meet their specific information needs-as opposed to educational information prescribed and delivered by healthcare providers.

To explore how people searched the internet, 97 participants recruited from the public waiting area in a cardiology clinic were asked to search MEDLINEplus and to search online as they would normally. The subjects were mostly white, educated, largely familiar with computers, and were used to searching the internet. They did so for different reasons: $12 \%$ did so routinely ("all the time"), $20 \%$ before or after a doctor's visit, 24\% when they experienced a particular problem ( $17 \%$ when a friend did); only $7 \%$ "rarely or never" searched for health information. The researchers measured success using four questions:

- Did you find what you were looking for?

- Do you think you will use the information?

- How do you rate your satisfaction with the search (on a scale of $1-10)$ ?

- Will you use the internet to find more information on this topic?

Comments about the information were more often negative than positive. Although some thought it was good because "I will be able to ask my doctor more specific questions", others felt the "information causes more questions because it is so confusing". People wanted more detail, less jargon, better organisation of information, and felt frustrated that "it takes a lot of effort to find one small thing".

The researchers observed participants when searching. They tended to "start browsing from the top of the content hierarchy" even though desired pages were several levels down. Consumers were not familiar with the organisation of MEDLINEplus.

The results present a certain paradox. Only $30 \%$ found what they were looking for and $58 \%$ said they would not use the information. Yet the mean satisfaction rating was 6.1 (on a scale of 1-10) and $74 \%$ said they would use the internet again. Interestingly, even when people failed to find what they were looking for, they still felt the answers to their questions "were out there". The problem was finding it.

Why is it that internet searches for health are so valued when the failure rate is so high? Based on interviews, the researchers argue that the answer is that "consumers have certain information needs that are even more difficult to address through other channels such as talking with clinicians". Because "speaking at length with a physician is often unfeasible, many consumers will instead look to the web in spite of the time, energy and frustrations involved".

The researchers suggest that "there are three ways to approach the problem: (1) make the queries more specific; (2) improve search engines so that they better match a query with appropriate targets; or (3) limit the search space". They conclude that, while "it is impractical to train millions of consumers to type in longer queries; however, it is possible to assist consumers in query construction by prompting for various refinements".

\ Zeng Q, Kogan S, Plovnick $M$, et al. Positive attitudes and failed queries: an exploration of the conundrums of consumer health information retrieval. Int $J$ Med Inf 2004;73:45-55.

How do the public prefer to receive health information from providers? A paper in Medical Decision Making studied how the public prefer to peruse information in order to make choices. Two studies were undertaken to compare reactions to information in tables and in narrative form. The authors hypothesised that, while the table format may be easier to read and therefore may facilitate understanding, the more personalised narrative format may allow patients to identify more with the health states described. The first group of patients $(n=14)$ had gastroentrological problems, the second $(n=30)$ were undergoing respiratory rehabilitation. Both groups were outpatients at McMaster University Hospital in Hamilton, Canada.

In each study the majority preferred the table format $(8 / 11$ in the first study and $23 / 30$ in the second). The table format was thought to be clearer, easier to read, and more straightforward to make comparisons. For many, the narrative format "had too many words". However, the smaller number of patients who preferred the narrative said it was "easier to understand" and "easier to read".

It may be that different groups require different forms of information. The researchers acknowledge that other patient groups such as younger patients may have different preferences. (The authors do not mention it, but it may be that different cultures have different preferences). It is acknowledged, however, that a "more attractive" narrative format might have had stronger appeal.

A Schunemann H, Stahl E, Austin P, et al. A comparison of narrative and table formats for presenting hypothetical health states to patients with gastrointestinal or pulmonary disease. Med Decis Making 2004;24:53-60.

Analysing resistance to evidence-based medicine - Professionals can face difficulties in using information and prefer to relate to it in different ways. Evidence-based medicine (EBM) is an increasingly popular usage model for information within medical informatics, but many professionals resist it.

Its proponents "continue to state that the goal of EBM is to save the practice of medicine from many of its major ills, including wide variations in clinical practice, use of unproven interventions, and failure to apply consistent practice guidelines. Opponents deny either the severity of these issues or that EBM can adequately address them, and dismiss EMB on the grounds of many philosophical and practical flaws."

Cohen et al from the Department of Medicine and Clinical Epidemiology at the School of Medicine, Oregon Health Science University categorise and evaluate the criticisms and limitations of EBM to gain a better understanding of the role it can most appropriately play in medical science and modern health care. After more than 10 years of debate and discussion about EBM, many areas of disagreement have emerged between supporters and detractors. The authors organise these into five main themes.

(1) EBM is based on empiricism, misunderstands or misrepresents the philosophy of science and is a poor philosophic basis for medicine.

(2) The EBM definition of evidence is narrow and excludes information important to clinicians.

(3) EBM is not evidence-based-that is, it does not meet its own empirical tests for efficacy.

(4) The usefulness of applying EBM to individual patients is limited.

(5) EBM threatens the autonomy of the doctor/patient relationship.

(1) A key criticism of EBM is that it "elevates experimental evidence to primary importance over other forms of evidence ... thus relegating to lesser importance the theory and the understanding of physiology and disease processes". The notion that EBM is "rational" and "objective" is questioned as "philosophers and 
scientists since the late 19th century [have argued that] making theory-free, objective observation is impossible ${ }^{\prime \prime}$. The authors say the touting of EBM as "a revolutionary new paradigm" is unfortunate and that those with this view are "disregarding the philosophy of science". They welcome the recent development whereby "some EBM proponents have backed off this position, and are now promoting EBM as a new tool available to augment the capabilities of the practicing clinician"

(2) "Evidence gathered by specific preferred methods is deemed better than evidence from other sources . . . giving the RCT the highest grade of evidence [even though] similarly designed RCTs researching the same question frequently disagree with each other". Criticisms of RCTs centre on three main points: (a) RCTs and metaanalysis have not been shown to be more reliable than other research methods, (b) the questions that EBM can answer are limited, and (c) EBM has failed to provide a means to integrate other non-statistical forms of medical information such as professional experience and patient specific factors.

(3) "Considering that EBM focuses on basing patient care on statistically valid clinical trials, it is somewhat surprising and ironic to find that there is no evidence (as defined by EBM) to back up this underlying assumption. There is little defence for a movement that does not hold to its own principles."

(4) "There are two problems in trying to apply ... statistical trends to individual patients. First, there is often a lack of studies relevant to the specific patient and intervention under consideration. Second, patients are individuals and not groups. There is no 'mean tendency' for a single patient."
(5) "Critics say that EBM ... may result in limiting the patient's right to choose what is best in his or her individual circumstances [and also] ... be used to prevent physicians from acting, holding them hostage and unable to use a treatment on a willing patient while waiting for appropriate statistical evidence". However, they note that "all the criticism of this type, as well as the defence, is based on conjecture".

The authors conclude that "none of the critics of EBM suggest that high quality evidence obtained by clinical epidemiological methods should be ignored in the context of patient care. But it is only one factor of many in a very complex context. EBM does not present a new philosophy of medicine, but it is instead a useful tool in making individual and group health care decisions and in discussing care with patients. The limitations of EBM must be carefully considered before current evidence is used to restrict patient access to health care, and outcomes must be watched closely for cases where the healthcare system has failed a patient because they were too unlike the average."

A Cohen A, Stavri PZ, Hersh W. A categorization and analysis of the criticisms of evidence-based medicine. Int J Med Inf 2004;73:35-43.

\section{$\mathrm{ECHO}$}

\section{Risk of infant death or stillbirth does not change with distance to hospital}

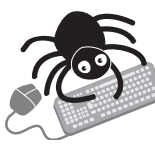

Please visit the Quality and Safety in Health Care website [www. gshc.com] for a link to the full text of this article. link between infant death or stillbirth and journey time to the nearest hospital has been refuted by statistics in northwest England covering more than four decades when used in a model providing a more realistic estimate of time travelled.

The data included all 287993 births in the whole of Cumbria, northwest England, for 1950-93, among which there were 4889 infant deaths and 4325 stillbirths. The deaths were grouped according to when they occurred after birth, and journey times to the nearest hospital at that time were computed with a geographical information system from grid references based on the postcodes of birth and hospital and data on the road network and location of built up areas between the two.

Over the entire period the odds of infant death were similar for early neonatal (0-6 days), neonatal (0-27 days), and postneonatal (28 days-1 year) deaths and all categories of journey times, from $0-17$ to over 35 minutes, after correction for demographic risk factorsbirth year, birth order, and multiple births. The same was true for decade by decade analysis. Stillbirths also showed no significant difference according to journey time. Median journey time to hospital was 12 (range $<1-70$ ) minutes.

There is little known in the UK about whether risk of infant death is affected by distance to nearest hospital. This large dataset confirms the results of a smaller study in West Cumbria a few years ago.

A Archives of Disease in Childhood 2004;89:232-234. 